\title{
Arachidonic acid induction of Rho-mediated transendothelial migration in prostate cancer
}

\author{
M Brown ${ }^{1}$, J-A Roulson ${ }^{1}$, C A Hart ${ }^{1}$, T Tawadros $^{1,3}$ and N W Clarke Ta, $^{*, 3}$ \\ ${ }^{1}$ Genito Urinary Cancer Research Group, Institute of Cancer Sciences, Paterson Building, The University of Manchester, \\ Manchester Academic Health Science Centre, Wilmslow Road, Manchester M20 4BX, UK and ${ }^{2}$ Department of Urology, Salford \\ Royal Hospital NHS Foundation Trust, Stott Lane, Salford M6 8HD, UK
}

Background: Bone metastases in prostate cancer (CaP) result in CaP-related morbidity/mortality. The omega-6 polyunsaturated fatty acid (PUFA) arachidonic acid (AA) and lipophilic statins affect metastasis-like behaviour in CaP cells, regulating the critical metastatic step of $\mathrm{CaP}$ migration to the bone marrow stroma.

Methods: Microscopic analysis and measurement of adhesion and invasion of CaP cells through bone marrow endothelial cells (BMEC) was undertaken with AA stimulation and/or simvastatin (SIM) treatment. Amoeboid characteristics of PC-3, PC3-GFP and DU-145 were analysed by western blotting and Rho assays.

Results: The CaP cell lines PC-3, PC3-GFP and DU-145 share the ability to migrate across a BMEC layer. Specific amoeboid inhibition decreased transendothelial migration (TEM). AA stimulates amoeboid characteristics, driven by Rho signalling. Selective knock-down of components of the Rho pathway (RhoA, RhoC, Rho-associated protein kinase 1 (ROCK1) and ROCK2) showed that Rho signalling is crucial to TEM. Functions of these components were analysed, regarding adhesion to BMEC, migration in $2 \mathrm{D}$ and the induction of the amoeboid phenotype by AA. TEM was reduced by SIM treatment of PC3-GFP and DU-145, which inhibited Rho pathway signalling.

Conclusions: AA-induced TEM is mediated by the induction of a Rho-driven amoeboid phenotype. Inhibition of this cell migratory process may be an important therapeutic target in high-risk CaP.

Prostate cancer $(\mathrm{CaP})$ metastasises to the bone marrow stroma (BMS) of the axial skeleton, causing major morbidity and mortality (Loberg et al, 2005). To spread to BMS, CaP cells must first escape the primary tumour, enter the peripheral blood and bind to endothelial surfaces in small vessels within the bone marrow. Migrating cells then invade through the endothelium into the BMS and coalesce/multiply to form a metastasis. This multistep metastatic process can be modelled in vitro using human primary BMS (Lang et al, 1998; Scott et al, 2001; Hart et al, 2005).

A gathering body of evidence has shown the importance of the omega-6 polyunsaturated fatty acid (PUFA) arachidonic acid (AA) in CaP; this is contained in bone marrow adipocytes (BM-Ad). It is strongly chemotactic for CaP cells (Brown et al, 2006) and its direct uptake from BM-Ad induces an aggressive, migratory phenotype
(Brown et al, 2006, 2010; Gazi et al, 2007). Clinically, AA intake has been associated with poorer $\mathrm{CaP}$ prognosis (Wynder et al, 1994) and an augmented risk of high-grade CaP (Chavarro et al, 2007; Szymanski et al, 2010). Intraprostatic AA levels are decreased within cancer tissue compared with adjacent normal tissue, suggesting that $\mathrm{AA}$ is being metabolised preferentially by $\mathrm{CaP}$ cells into pro-tumoral eicosanoids (Schumacher et al, 2011). We recently showed that this AA-induced behaviour was regulated by the ligand-independent activity of Ephrin receptor A2 (EphA2) (Tawadros et al, 2012).

Further lipid-based studies have linked lipid metabolism and its regulation by statins with altered aggressiveness in CaP. Epidemiological studies have reported lower rates of $\mathrm{CaP}$ progression in patients taking statins (Platz et al, 2006; Platz, 2008; Breau et al, 2010;

*Correspondence: Professor NW Clarke; E-mail: noel.clarke@srft.nhs.uk
3Joint senior authors.

Received 18 August 2013; revised 23 January 2014; accepted 28 January 2014; published online 4 March 2014 
Murtola et al, 2010) and these patients had less aggressive histological features (Gutt et al, 2010; Loeb et al, 2010). The basis for these observations is poorly understood but the effects probably relate to the pleiotropic actions of 3-hydroxy-3-methylglutarylCoA (HMG-CoA) inhibitors on cellular behaviour, cell-cell interaction and cellular motility in relation to lipid metabolism (Roy et al, 2011). We observed that lipophilic statins reduce the migration and colony formation of $\mathrm{CaP}$ in $\mathrm{BMS}$ co-culture, an effect mediated through the inhibition of GGPP production, which is required for the post-translational modification of proteins such as GTPases (Brown et al, 2012).

The phenomenon of epithelial-mesenchymal transition (EMT) (Kopfstein and Christofori, 2006) has been studied intensively in relation to metastasis. There are two phenotypes associated with EMT and invasion: amoeboid and mesenchymal. While EMT is structured, following extensive alterations in gene transcription, transition between the mesenchymal and amoeboid phenotype occurs rapidly, involving morphological transition, with concomitant changes in migratory behaviour arising in response to microenvironmental changes. Amoeboid invasion is promoted by the Rho/Rho-associated protein kinase (ROCK) signalling pathway (Pankova et al, 2010), which enhances the contractility of cells, enabling them to squeeze through gaps in the ECM, adapting their shape to the pre-existing spaces, or exerting forces that deform the surrounding extracellular matrix (Pankova et al, 2010). The intracellular tension maintained by cortical actin-myosin during this process results in membrane blebbing, contributing to cellular motility (Pankova et al, 2010).

Rho family GTPases affect cell motility and polarity (Jaffe and Hall, 2005; Boureux et al, 2007) and are implicated in cancer progression and metastasis (Sahai and Marshall, 2002). The Rho subfamily includes the isoforms RhoA and RhoC, which are $84 \%$ identical in sequence; most differences are concentrated near the $\mathrm{C}$ terminus (Wheeler and Ridley, 2004). Both RhoA and RhoC can induce stress fibres (Aktories and Just, 2005) but several lines of evidence indicate that the isoforms have different functions. RhoC is selectively upregulated during EMT (Dietrich et al, 2009), which occurs in some cancers (Thiery, 2002). Multiple targets have been identified for Rho proteins (Jaffe and Hall, 2005) but it is not clear whether the isoforms act selectively through different or common downstream targets to mediate specific responses. Where tested, the isoforms generally bind to the same targets in vitro; for example, both RhoA and RhoC bind to the Rho-associated kinases ROCK1 and ROCK2 (Leung et al, 1996). However, RhoC but not RhoA has been suggested to bind specifically to the formin FMNL2 (Kitzing et al, 2010).

Herein, we studied Rho function during transendothelial migration (TEM) and its regulation by putative modulators of $\mathrm{CaP}$ aggression.

\section{MATERIALS AND METHODS}

Materials. All tissue culture, inhibitors and general reagents were purchased from Sigma-Aldrich (Poole, UK), except AA (MP Biomedicals, London, UK), which was emulsified at $10 \mathrm{mg} \mathrm{ml}^{-1}$ in methyl- $\beta$-cyclodextrin. Hygromycin B and foetal calf serum (FCS) were from PAA Laboratories (Yeovil, UK). Matrigel and $8 \mu \mathrm{m}$ FluoroBlok cell-culture inserts were from BD Biosciences (Oxford, UK).

Antibodies were from Cell Signalling Technology (New England BioLabs (UK) Ltd, Hitchin, UK) except GAPDH, which was from Abcam (Cambridge, UK). Y-27632, Rac-1 inhibitor, GM6001, H1152 and blebbistatin were from Merck Biosciences (Nottingham, UK). All On-Target Plus Smartpool siRNAs were from Dharmacon, Thermo Fisher Scientific (Fermentas GmbH, St Leon,
Germany). Protease and phosphatase inhibitor cocktail were purchased from Roche (Sussex, UK).

Cell culture. All cell lines were verified by the Paterson Institute for Cancer Research tissue typing service by genotyping and cultured as described previously (Hart et al, 2005; Tawadros et al, 2012). VCaP and DU-145 were grown in RPMI-1640 with $10 \%$ FCS, $2 \mathrm{~mm}$ L-Glutamine, with the addition of $10 \mathrm{~mm}$ sodium pyruvate and $100 \mathrm{~mm}$ HEPES for VCaP.

Transfection. PC3-GFP and DU-145 cells were transfected with 600 ng of siRNA using Dharmafect 2 (Thermo Fisher Scientific) in antibiotic-free media according to the manufacturers' instructions. siRNA knock-down of target protein was confirmed by western blotting.

Western blotting. Cell lysates, total protein content and western blot procedures were performed as previously described (Tawadros et al, 2012).

Rho assay. Briefly, $8 \times 10^{5}$ PC3-GFP cells were rinsed in ice-cold PBS and lysed in $900 \mu \mathrm{l}$ MLB buffer with protease inhibitor $(25 \mathrm{~mm}$ HEPES, pH 7.5, $150 \mathrm{~mm} \mathrm{NaCl}, 1 \%$ Igepal CA-630, $10 \mathrm{~mm} \mathrm{MgCl}_{2}$, $1 \mathrm{~mm}$ EDTA, $10 \%$ glycerol). Lysates were cleared of insoluble cell debris by centrifugation $\left(5 \mathrm{~min}, 14000 \mathrm{~g}, 4^{\circ} \mathrm{C}\right.$ ) and incubated $\left(45 \mathrm{~min}\right.$ at $4{ }^{\circ} \mathrm{C}$ ), with $20 \mu \mathrm{g}$ of a Rhotekin Rho Binding DomainGST tagged fusion protein bound to glutathione-agarose (Millipore UK Ltd, Watford, UK). After three washes in MLB, agarose beads were resuspended in western blot loading buffer.

Invasion assay. Invasion assays with PC-3, PC3-GFP, DU-145, $\mathrm{VCaP}, \mathrm{LNCaP}$ and PNT2-C2 cells were performed according to Brown et al (2006) and Hart et al (2005), respectively.

Adhesion assay. Adhesion assays with PC3-GFP were performed as described (Tawadros et al, 2012). Adhesion assays with DU-145 were performed using a variation of Scott et al (2001): $4 \times 10^{4}$ serum-starved $(20 \mathrm{~h}) \mathrm{DU}-145$ cells were added to a confluent bone marrow endothelial cell (BMEC) layer with or without AA $(10 \mu \mathrm{M})$. After incubation at $37^{\circ} \mathrm{C}$ for $15 \mathrm{~min}$, non-adherent cells were removed by $2 \times 200 \mu$ l PBS washes and fixed in methanol-acetone. Cytokeratin staining was performed as previously described (Scott et al, 2001).

Migration assay. Cells were cultured in 6-well plates until $90 \%$ confluence before serum starvation in RPMI media for $16 \mathrm{~h}$. Photographs were taken just after wells were scratched using a thin sterile pipette tip and $24 \mathrm{~h}$ later, after the addition of medium without serum, with AA or simvastatin (SIM). PC3-GFP was also transfected $48 \mathrm{~h}$ before with siNT, siRhoA, siRhoC, siROCK1 and siROCK2, respectively, and DU-145 with siNT, siRhoA, and siRhoC, respectively.

Time lapse video microscopy invasion studies. Confluent BMEC layers in BMS conditioned media (BMSCM), gassed with $5 \% \mathrm{CO}_{2}$ in air, in $12.5 \mathrm{~cm}^{2}$ tissue culture flask were imaged at two frames every $20 \mathrm{~s}$ for $3 \mathrm{~h}$ (total magnification $\times 100$ ) before the addition of $6.25 \times 10^{5}$ PC-3, PC-3-GFP or PNT2-C2 cells. The co-culture was imaged at two frames every $20 \mathrm{~s}$ for $24 \mathrm{~h}$ at $37^{\circ} \mathrm{C}, 5 \% \mathrm{CO}_{2}$ in air. Images were edited using Pinnacle Studio 9 (Corel Corporation, Ottawa, ON, Canada). Inhibitors were added to the prostate cell suspension before addition to the BMEC monolayer.

Electron microscopy. PC-3 cells were added to confluent BMEC cultured on fibronectin-coated $5 \times 5 \mathrm{~mm}$ Thermanox plastic coverslips for $5 \mathrm{~h}$ before washing $2 \times$ in Sorenson's Buffer and overnight fixation in 3\% glutaraldehyde in Sorenson's buffer at $4{ }^{\circ} \mathrm{C}$. Coverslips were washed in Sorenson's buffer, post-fixed with 1\% osmium tetroxide (Agar Scientific, Stansted, UK) in Sorensen's buffer before washing in double distilled water and dehydrating through sequential solutions of ethanol (30\%, 50\%, 70\%, 95\%, 95\%, 
$100 \%$, and 100\%). Ethanol was exchanged for Arklone (Taab Laboratory Supplies, Reading, UK) before critical point drying. Coverslips were sputter coated with $4 \mathrm{~nm}$ chromium before mounting onto aluminium stubs (Agar Scientific) using Acheson ElectroDAG $1415 \mathrm{M}$ conducting adhesive (Agar Scientific) and sputter coating with $8 \mathrm{~nm}$ platinum-palladium $80 / 20$. Coverslips were imaged using an ABT DS130-F dual stage field emission scanning electron microscope (International Scientific Instruments, Milpitas, CA, USA) at an accelerating voltage of $20-30 \mathrm{kV}$.

Statistics. All values are presented as mean \pm s.e.m. All assays were compared using Student's two-tailed $t$-test with significance set at $P<0.05$.

\section{RESULTS}

AA induces an amoeboid phenotype. Invasion assays towards human primary BMS, AA and tissue culture plastic (TCP) were used to evaluate TEM abilities of different CaP cell lines. Only PC3 , the sub-clone PC3-GFP and to a lesser extent DU-145 cells exhibited the ability to migrate across a confluent human BMEC monolayer and synthetic basement membrane (Matrigel) (Figure 1A). VCaP, LNCaP and PNT2-C2 were unable to migrate across an endothelial layer (Figure 1A). Time-lapse microscopy of PC-3 and PC3-GFP transmigration across a BMEC layer in presence of BMEC conditioned media showed that cells migrated rapidly across the endothelial barrier (Figure 1B; Supplementary Figure 2; Supplementary Video 2A).

Incubation of PC3-GFP cells with the omega-6 PUFA AA, a potent stimulator of $\mathrm{CaP}$ cell invasion, induced morphological changes associated with an amoeboid phenotype. The percentage of rounded PC3-GFP cells increased after $3 \mathrm{~h}$ AA incubation $(P \leqslant 0.01)$ (Figure 2A; Supplementary Figure 1) and this was associated with increased RhoA and RhoC activity (2.2 and 2.3-fold, respectively; Figure 2B). The Rho signalling pathways induce stress fibres that are associated with increased levels of P-MLC2 (myosin II light chain) (Lammermann and Sixt, 2009). Western blot analysis of PC3-GFP cells incubated with AA $(10 \mu \mathrm{M})$ showed increased P-MLC2 levels after $60 \mathrm{~min}(P \leqslant 0.01)$ (Figure 2C). AA was also shown to activate the same pathway and subsequent phosphorylation in the less invasive DU-145 cell line. We have previously reported that AA acts though the ligand-independent activation of Ephrin A2 via phosphorylation of Akt (Tawadros et al, 2012). As with the PC-3 cell line, Figure 2D shows that AA induces the phosphorylation of both Akt and MLC2 in DU-145 cells. As a control, pre-incubation of PC3-GFP cells with the ROCK inhibitor Y27632 $(40 \mu \mathrm{M})$ decreased P-MLC2 levels at baseline and after AA stimulation (Figure 3B). Additionally, electron microscopy revealed that PC-3 cells bound to junctional areas of confluent BMEC layer in the presence of bone marrow conditioned media became morphologically round, with a blebbing form consistent with an amoeboid phenotype before TEM (Figure 2E; Supplementary Figure 2B). PC-3 pre-incubation with $40 \mu \mathrm{M}$ Y27632 (ROCK inhibitor), or $75 \mu \mathrm{M}$ blebbistatin (non-muscle myosin II inhibitor), demonstrated that Rho/ROCK activity and actin-myosin contractility are involved in the transmigration process (Figure $2 \mathrm{~F}$ ), by comparison with Rac signalling ( $75 \mu \mathrm{M}$ Rac-1 inhibitor) or metalloproteinase inhibition (20 $\mu \mathrm{M}$ GM6001) (Figure 2F). Y27632 and blebbistatin had no effects on the dextran permeability of the BMEC monolayer (Supplementary Figure 2C).

TEM towards AA is mediated by the Rho signalling pathway. The selective effect of the Rho/ROCK signalling pathway during TEM was determined by comparing invasion through a Matrigel synthetic basement membrane with invasion towards AA through a confluent BMEC monolayer on Matrigel. Pre-incubation of PC3GFP cells with the ROCK inhibitor Y27632 $(40 \mu \mathrm{M})$ or H1152 $(5 \mu \mathrm{M})$ (Supplementary Figure 3D) did not affect invasion through Matrigel (Figure 3A, left panel). By contrast, ROCK inhibition significantly decreased TEM (Figure 3A, right panel) towards AA through a BMEC layer. Y27632 did not affect viability (Supplementary Figure 3A) but it induced an amoeboid to mesenchymal phenotypic change (Supplementary Figure 3B). To further characterise the Rho signalling pathway, selective siRNA knock-down of RhoA, RhoC, ROCK1 and ROCK2 was performed. Knock-down of RhoA, RhoC, ROCK1 and ROCK2 in PC3-GFP
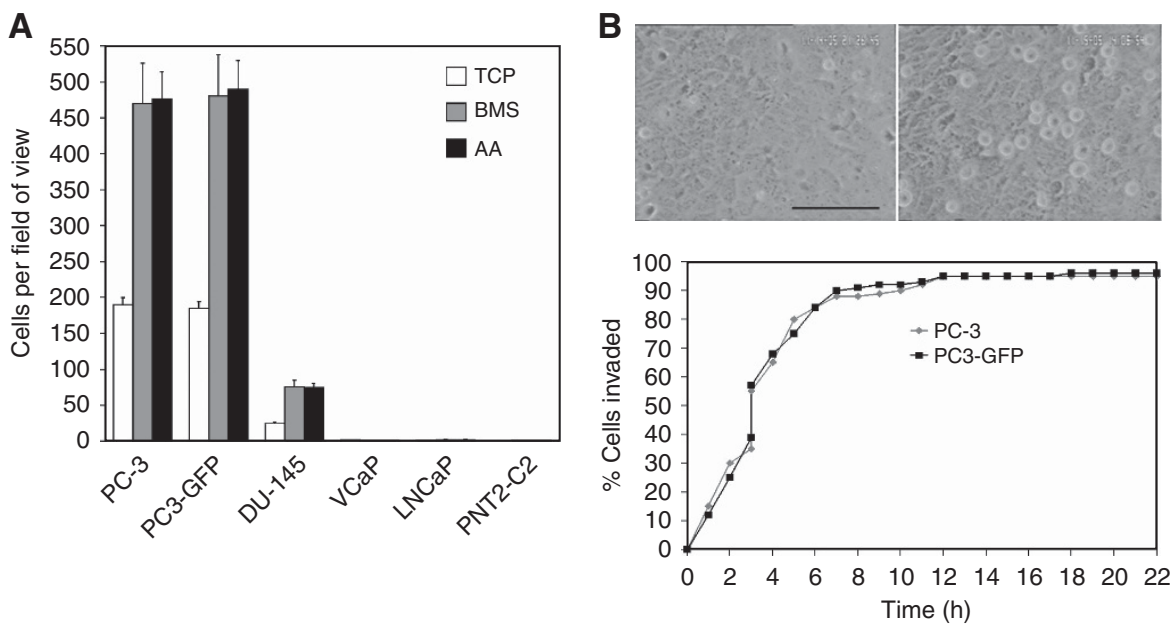

Figure 1. Ability of PC-3 cells to invade a BMEC layer. (A) Invasion assays were performed using cell-culture inserts $(8 \mu \mathrm{m}$ pore size) with a barrier of a confluent layer of BMEC above a synthetic basement membrane (Matrigel). $1 \times 10^{5}$ PC-3, PC3-GFP, DU-145, LNCaP, VCaP and PNT2-C2 cells were added to the top of the inserts and allowed to invade for $18 \mathrm{~h}$ towards tissue culture plastic (TCP), bone marrow stroma (BMS) or AA (10 $\mu \mathrm{M})$. Data are means \pm s.e.m. of triplicate samples from two independent experiments. (B) Live-cell invasion studies using time lapse video microscopy. BMEC monolayer before addition of PC-3 cells (left). BMEC monolayer with PC-3 cells present as rounded cells on top of BMEC 20 min after addition of PC-3 cells (right). Scale bar represents $100 \mu \mathrm{m}$. PC-3 or PC3-GFP cells were seeded onto a confluent BMEC monolayer, in the presence of $\mathrm{AA}(10 \mu \mathrm{M})$, and observed using time lapse video microscopy. The cumulative percentage of cells completing invasion plotted against time is shown. Diamond: PC-3 cells. Square: PC3-GFP cells. 
A

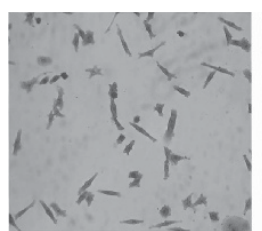

Ctrl

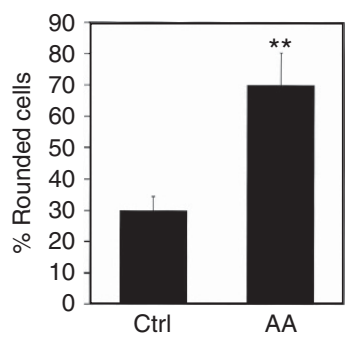

C

Time (min)

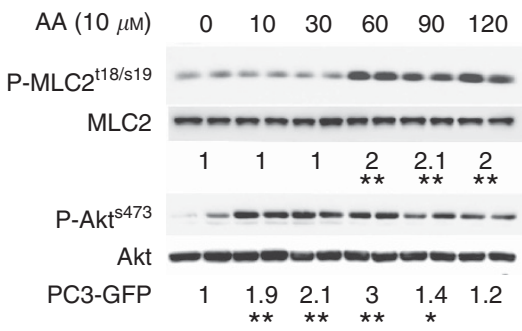

E

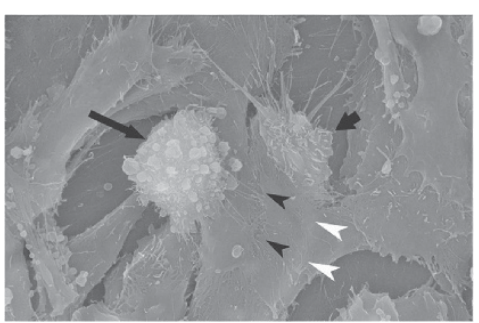

B

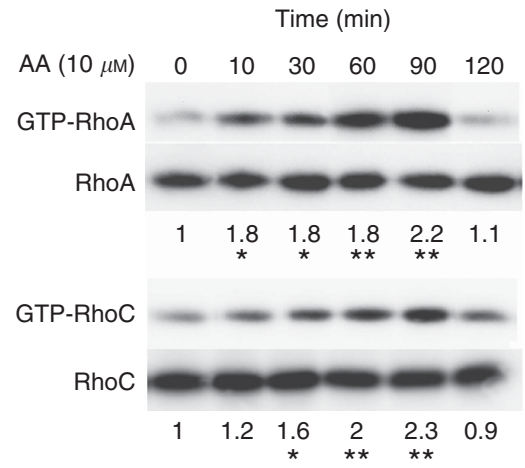

D
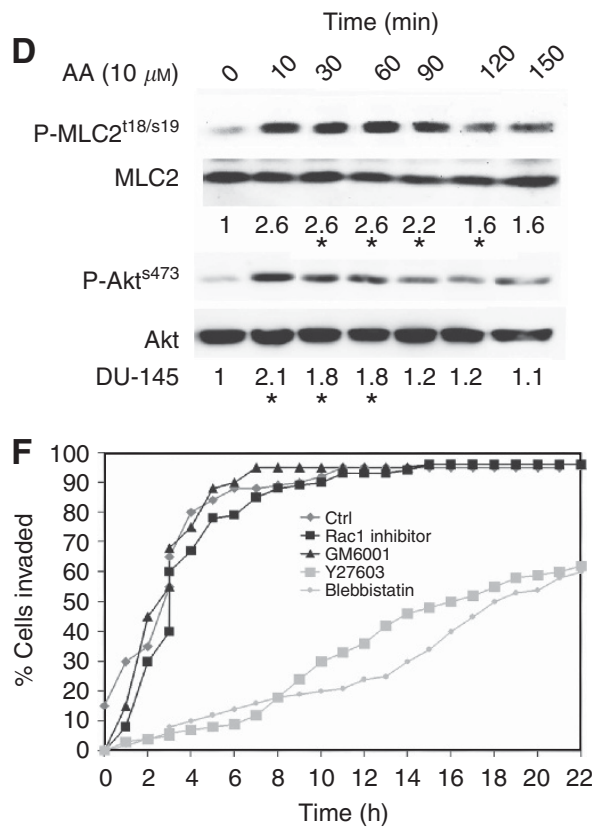

Figure 2. Arachidonic acid induces RhoA and C activities in PC3-GFP. (A) Microscopic analysis of PC3-GFP cells incubated with AA (10 $\mu \mathrm{M})$. Serumstarved PC3-GFP cells were incubated with AA $(10 \mu \mathrm{m})$ or the vehicle only during $3 \mathrm{~h}$. Cells were then fixed, stained with crystal violet and photographed. Lower panel: 150 cells were counted per condition and the percentage of rounded cells counted. Data are means \pm s.e.m. of duplicate samples from two independent experiments. ${ }^{*} P<0.01$ vs no AA. (B) Lysates of PC3-GFP cells incubated with AA (10 $\left.\mu \mathrm{M}\right)$ at different times were subjected to Rho assays. Levels of GTP-RhoA and GTP-RhoC were compared with total levels of RhoA and RhoC, respectively. Figure is representative of at least three individual experiments done in duplicate. Quantification of densitometric values of GTP-RhoA and GTP-RhoC in AA-stimulated PC3-GFP samples compared with total levels of RhoA and RhoC, respectively. ${ }^{*}<0.05,{ }^{* \star} P<0.01$ vs no AA. Densitometric values of controls without incubation with AA were set at one. (C) Lysates of PC3-GFP cells incubated with AA (10 $\mu$ M) at different times were subjected to western blotting. Phosphorylation levels of $\mathrm{Akt}^{\mathrm{s} 473}$ and $\mathrm{MLC}^{\mathrm{t18} / \mathrm{s} 19}$ were compared with total levels of Akt and MLC2, respectively. Figure is representative of three separate experiments done in duplicate. Quantification of densitometric values of phosphorylated $\mathrm{Akt}^{5473}$ and MLC $\mathrm{C}^{\mathrm{t} 18 / \mathrm{s} 19}$ in AA-stimulated PC3-GFP samples was compared with the total levels of Akt and MLC, respectively. ${ }^{\star} P<0.05,{ }^{\star \star} P<0.01$ vs no AA. Densitometric values of controls without incubation with AA were set at one. (D) P-MLC2 ${ }^{\mathrm{t} 18 / \mathrm{s} 19}$, MLC2, P-Akt and Akt content was analysed by western blotting in lysates of DU-145 cells incubated with AA at different times. Levels of P-Akt and P-MLC2 were compared with total levels of Akt and MLC2, respectively. Figure is representative of two separate experiments done in duplicate. ${ }^{\star} P \leqslant 0.05$ vs Ctrl (without $A A$ ). Densitometric values of controls were set at one. (E) SEM imaging of PC-3 transendothelial migration. SEM images of a BMEC monolayer $5 \mathrm{~h}$ after addition of PC-3 cells. A rounded and blebbing cell (long arrow), and part of a pseudopodium (short arrow) appears to be extending from underneath flattened BMEC in the area of a BMEC cell-cell junction (arrowheads). The edges of the under lapping cell can be seen as an area where BMEC appear to be lifted (white arrowheads). Magnification: $\times 1500$. (F) Migration of PC3-GFP cells through a BMEC monolayer in the presence of motility inhibitors. PC3-GFP cells were seeded onto a confluent monolayer of BMEC and observed for $24 \mathrm{~h}$ in the time lapse video microscopy unit. In the control experiment, 25 cells were followed, 37 in the Y27632 experiment, 28 for blebbistatin, 29 for Rac1 inhibitor and 22 for GM6001.

cells did not modulate AA-induced invasion through Matrigel (Figure 3C, left panel) but knock-down of all four induced a significant reduction in AA-induced invasion across the BMEC layer $(P \leqslant 0.01)$ (Figure $3 \mathrm{C}$, right panel). Moreover, combinations of RhoA/RhoC and ROCK1/ROCK2 knock-down showed no significant change in the inhibitory effect (Supplementary Figure 4). Selective knock-down in DU-145 (Figure 3D; Supplementary Figure 6) showed, as in PC3-GFP, that both RhoA and RhoC are involved in DU-145 TEM (Figure 3D, left and right panels). 
A

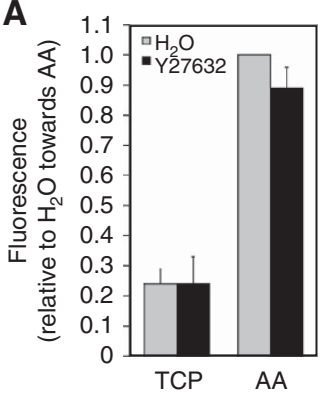

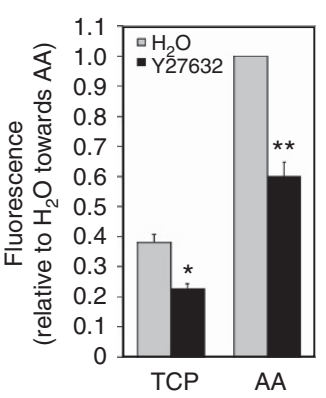

B

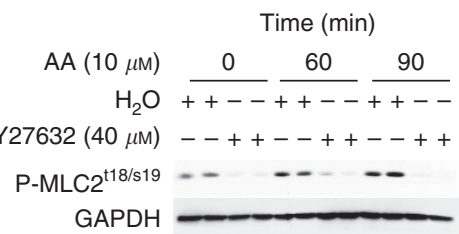

C
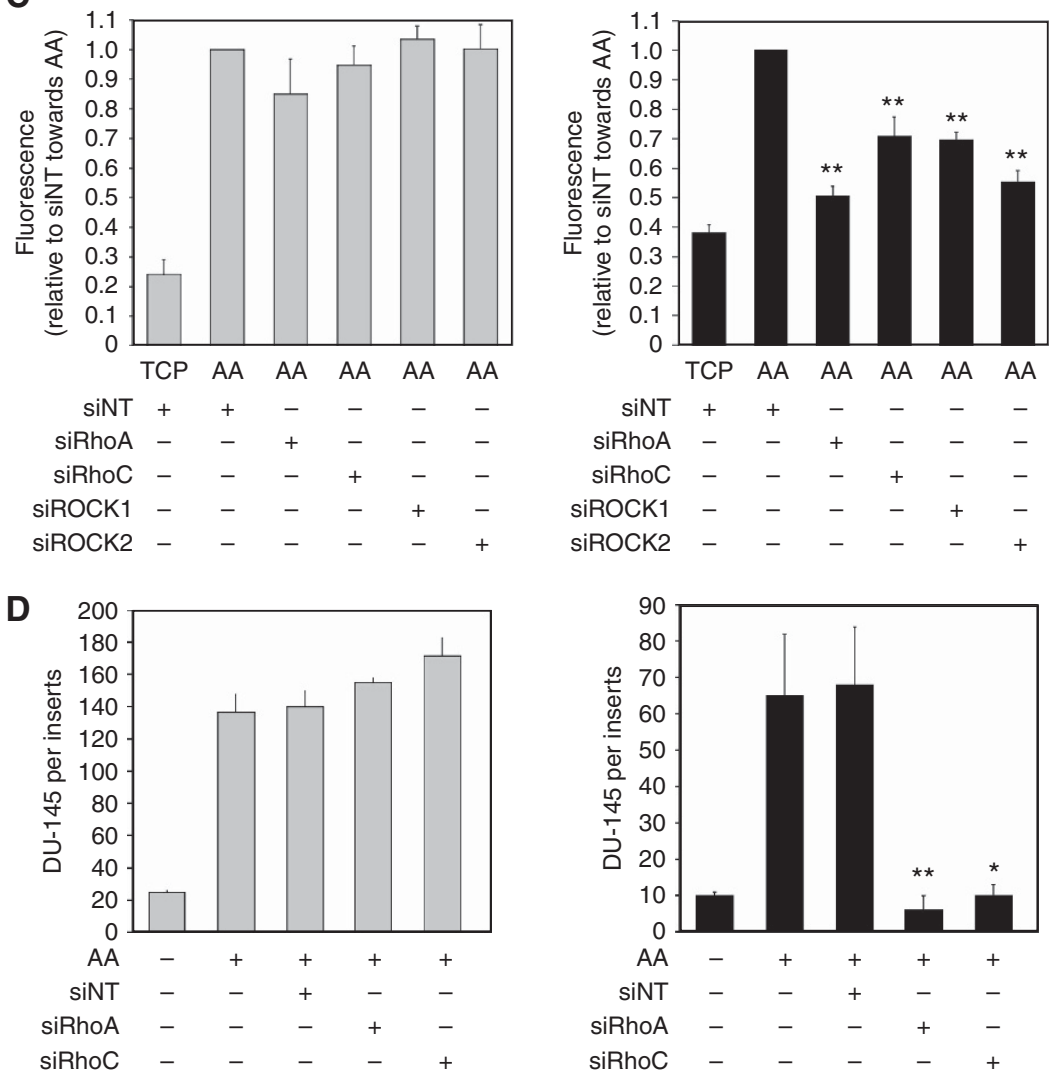

Figure 3. Rho signalling controls transendothelial migration towards AA. (A) Invasion assays with PC3-GFP cells were performed using cell-culture inserts ( $8 \mu \mathrm{m}$ pore size) coated by a synthetic basement membrane (Matrigel) (left panel) or culture inserts ( $8 \mu \mathrm{m}$ pores) coated by a layer of BMEC above a synthetic basement membrane (Matrigel) (right panel). $2 \times 10^{5}$ PC3-GFP cells pre-incubated with Y27632 $(40 \mu \mathrm{M})$ or the vehicle $\left(\mathrm{H}_{2} \mathrm{O}\right)$ for $30 \mathrm{~min}$ were added to the top of the inserts and allowed to invade towards AA $(10 \mu \mathrm{M})$ for $18 \mathrm{~h}$. Levels of invasion are proportional to fluorescence detected by a bottom reading BMG FLUOstar OPTIMA plate reader at 488/520 nm (excitation/emission filter). Data represent means \pm s.e.m. of three separate experiments (in triplicate). ${ }^{*} P<0.05,{ }^{\star} P \leqslant 0.01$ vs no Y27632 towards TCP or AA, respectively. (B) P-MLC2 levels in PC3-GFP cells incubated with AA and/or the ROCK inhibitor Y27632. Lysates of serum-starved PC3-GFP cells incubated with AA (10 $\mu \mathrm{m}) \pm \mathrm{Y} 27632(40 \mu \mathrm{M}, 1 \mathrm{~h}$ pre-incubation) at different times were subjected to western blotting. Phosphorylation levels of MLC ${ }^{\text {t18/s19 }}$ were compared with GAPDH. Figure is representative of two separate experiments done in duplicate. (C) Invasion assays with PC3-GFP cells were performed using cell-culture inserts ( $8 \mu \mathrm{m}$ pore size) coated by a synthetic basement membrane (Matrigel) (left panel) or culture inserts ( $8 \mu \mathrm{m}$ pores) coated by a layer of BMEC above a synthetic basement membrane (Matrigel) (right panel). $2 \times 10^{5}$ PC3-GFP cells were added to the top of the inserts and allowed to invade towards AA $(10 \mu \mathrm{m})$ for $18 \mathrm{~h}$. PC3-GFP cells were transfected with specific RhoA (siRhoA), RhoC (siRhoC), ROCK1 (siROCK1), ROCK2 (siROCK2) siRNA pools, respectively, or with non-targeted siRNA (siNT) 2 days before the invasion assays. Data represent means \pm s.e.m. of three separate experiments (in triplicate). ${ }^{\star \star} P \leqslant 0.01 \mathrm{vs}$ siNT towards AA. (D) Invasion assays with DU-145 cells were performed using cell-culture inserts (8 $\mu \mathrm{m}$ pore size) coated by a synthetic basement membrane (Matrigel) (left panel) or culture inserts ( $8 \mu \mathrm{m}$ pores) coated by a layer of BMEC above a synthetic basement membrane (Matrigel) (right panel). $2 \times 10^{5} \mathrm{DU}-145$ cells were added to the top of the inserts and allowed to invade towards AA (10 $\mu \mathrm{M}$ ) for 18 h. DU-145 cells were transfected with specific RhoA (siRhoA) and RhoC (siRhoC) siRNA pools, respectively, or with non-targeted siRNA (siNT) 2 days before the invasion assays. Data represent means \pm s.e.m. of three separate experiments (in triplicate). ${ }^{\star} P<0.05,{ }^{\star \star} P \leqslant 0.01 \mathrm{vs}$ siNT towards AA. Cells were counted after staining with either crystal violet or CK immunostaining.

TEM is associated with several steps starting with the adhesion of cells to the endothelium, migration to the intercellular junction between endothelial cells and finally endothelial cell retraction, facilitating transmigration of the cell body with detachment and retraction of the trailing edge (Hart et al, 2005). Adhesion assays with PC3-GFP pre-incubated with $40 \mu \mathrm{M}$ Y27632 showed that adhesion induced by AA is decreased by ROCK inhibition compared with PC3-GFP cells incubated with the vehicle only 
A

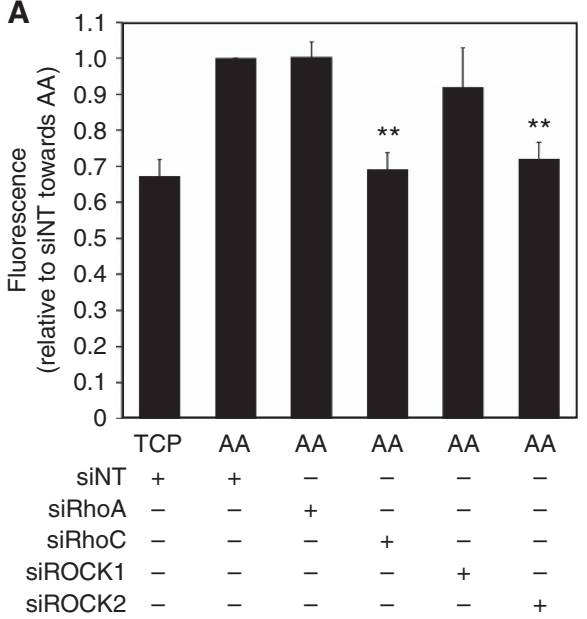

C

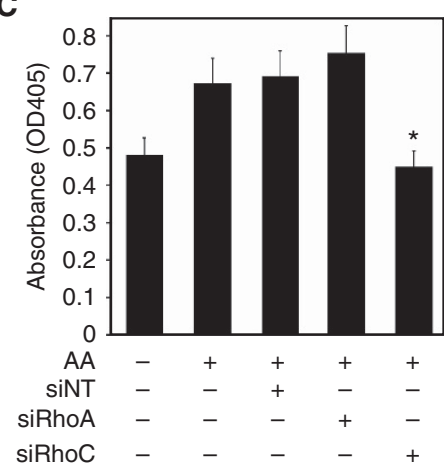

E

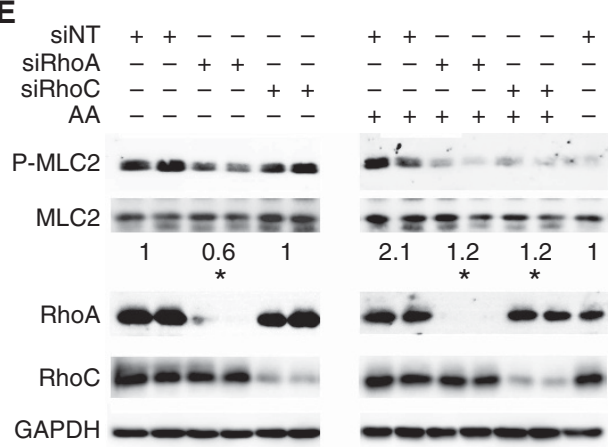

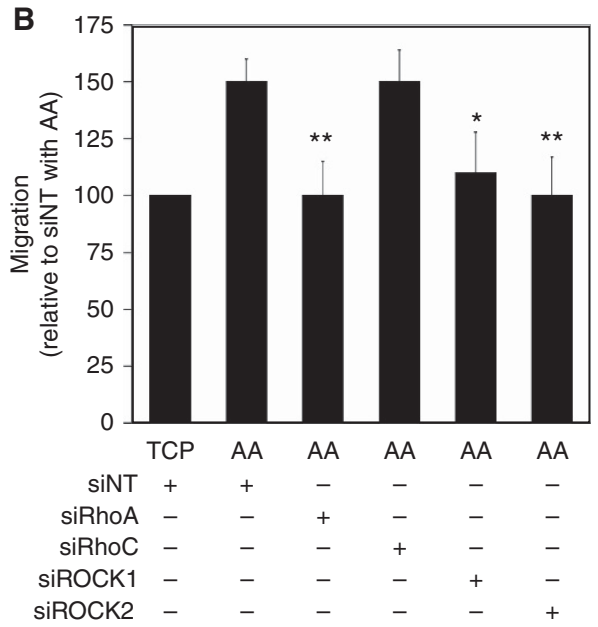
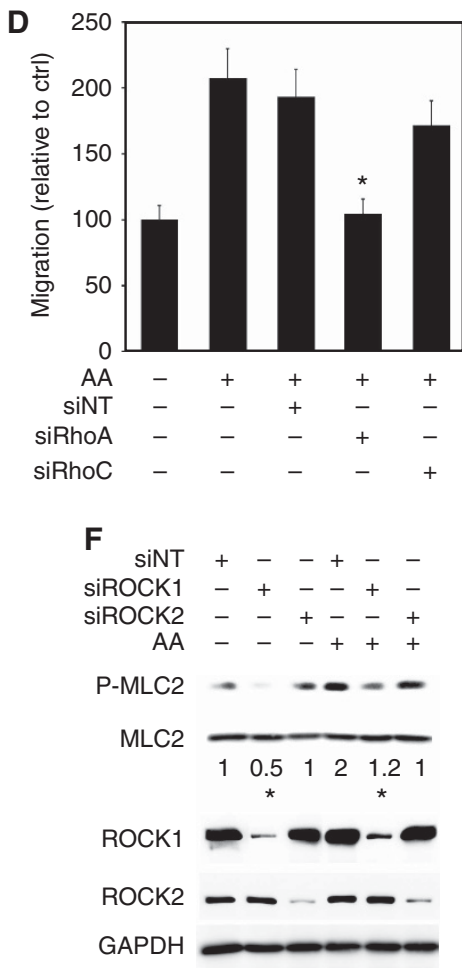

Figure 4. AA-stimulated transendothelial migration is mediated by specific Rho GTPase signalling. (A) Adhesion assays were performed using PC3-GFP with or without AA $(10 \mu \mathrm{M})$ added at the beginning of the assay. $4 \times 10^{4}$ PC3-GFP cells were then incubated for 8 min with a confluent layer of BMEC plated in 96 wells. Wells were washed twice in PBS. Levels of adhesion are proportional to fluorescence detected by a bottom reading BMG FLUOstar OPTIMA plate reader at $488 / 520 \mathrm{~nm}$ (excitation/emission filter). PC3-GFP cells were transfected with specific RhoA (siRhoA), RhoC (siRhoC), ROCK1 (siROCK1), ROCK2 (siROCK2) siRNA pools, respectively, or with non-targeted siRNA (siNT) 2 days before the adhesion assays. Data represent means \pm s.e.m. of three separate experiments. ${ }^{\star} P \leqslant 0.01$ vs siNT with AA. (B) Migration assays were performed using PC3-GFP with or without AA (10 $\mu \mathrm{m})$. PC3-GFP cells were transfected for $48 \mathrm{~h}$ with specific RhoA (siRhoA), RhoC (siRhoC), ROCK1 (siROCK1), ROCK2 (siROCK2) siRNA pools, respectively, or transfected with non-targeted siRNA (siNT) $48 \mathrm{~h}$ before the assay. Data represent means \pm s.e.m. of three separate experiments. ${ }^{\star} P \leqslant 0.05,{ }^{*} P \leqslant 0.01$ vs siNT with AA. (C) Adhesion assays to BMEC over 15 min \pm AA (10 $\left.\mu \mathrm{M}\right)$ were undertaken using DU-145 cells. These were transfected with specific RhoA (siRhoA) and RhoC (siRhoC) siRNA pools, respectively, or with non-targeted siRNA (siNT) 2 days before the adhesion assays. Data represent means \pm s.e.m. of three separate experiments. ${ }^{\star} P \leqslant 0.05$ vs no SIM with AA. (D) Migration assays were performed using DU-145 \pm AA $(10 \mu \mathrm{m})$. DU-145 cells were transfected with specific RhoA (siRhoA) and RhoC (siRhoC) siRNA pools, respectively, or with non-targeted siRNA (siNT) 2 days before the migration assays. Data represent means \pm s.e.m. of two separate experiments. ${ }^{*} P \leqslant 0.05$ vs no SIM with AA. (E) P-MLC2 ${ }^{\mathrm{t18} / \mathrm{s} 19}, \mathrm{MLC2}$, RhoA and RhoC content was analysed by western blotting in lysates of PC3-GFP cells transfected for $48 \mathrm{~h}$ with specific RhoA (siRhoA) and RhoC (siRhoC) siRNA pools, respectively, or transfected with non-targeted siRNA (siNT) and incubated with or without $A A(10 \mu \mathrm{M})$ for $75 \mathrm{~min}$. Figure is representative of three separate experiments. ${ }^{*} P \leqslant 0.05 v s$ siNT \pm incubation with $A A$. Densitometric values of siNT \pm incubation with $A A$ were set at one. (F) P-MLC2 ${ }^{t 18 / s 19}, M L C 2, R O C K 1$ and ROCK2 content was analysed by western blotting in lysates of PC3-GFP cells transfected for $48 \mathrm{~h}$ with specific ROCK1 (siROCK1) and ROCK2 (siROCK2) siRNA pools, respectively, or transfected with non-targeted siRNA (siNT) and incubated $\pm A A(10 \mu \mathrm{M})$ for 75 min. Figure is representative of three separate experiments. ${ }^{*} P \leqslant 0.05$ vs siNT without or with incubation with AA. Densitometric values of siNT with or without incubation with AA were set at one. 
$(P \leqslant 0.01)$ (Supplementary Figure 4$)$. Selective knock-down of RhoC and ROCK2 in PC3-GFP also decreased adhesion to BMEC induced by AA compared with control $(P \leqslant 0.01)$ (Figure $4 \mathrm{~A}$ ). Conversely, knock-down of RhoA and ROCK1 had no significant effect on AA-induced binding of PC3-GFP cells to the BMEC cells (Figure 4A).

Migration in 2D, evaluated by scratch assay, showed that migration of PC3-GFP cells incubated with AA $(10 \mu \mathrm{M})$ increased by $50 \%(P<0.01)$. Furthermore, siRNA knock-down of RhoA, ROCK1 and ROCK2 in PC3-GFP decreased AA-induced migration (Figure 4B). The involvement of both RhoC in AA-induced BMEC adhesion and RhoA in AA-induced migration was also demonstrated in the DU-145 cell line (Figure 4E and F).

Stress fibre formation, associated with the amoeboid phenotype, was measured by determining P-MLC2 levels. In PC3-GFP samples, selective knock-down of RhoA and ROCK1 decreased P-MLC2 levels at basal state compared with control PC3-GFP cells transfected with the control siRNA (Figure 4C and D). By contrast, lysates of PC3-GFP incubated with AA $(10 \mu \mathrm{M})$ revealed that RhoA, RhoC, ROCK1 and ROCK2 are required for the phosphorylation of MLC2 following AA induction (Figure 4E and F). P-MLC2 levels regulated by the different Rho signalling isoforms correlated with PC3-GFP phenotype (Supplementary Figure 5).

Effect of SIM on migrational behaviour and Akt/Rho signalling. We recently showed that statins inhibit invasion of PC-3 cells through a BMEC layer towards human primary BMS by blocking geranylgeranyl pyrophosphate (GGPP) production, which is required for the post-translational modification of proteins such as the Rho family of GTPases (Brown et al, 2012). Statins should, theoretically, prevent AA-induced mesenchymal to amoeboid switching by inhibiting the post-translational modification of the Rho GTPases by GGPP, thereby preventing Rho being targeted to the membrane to induce its signalling cascade. We assessed the effect of the statin SIM on the ability of AA to induce PC3-GFP invasion, TEM, adhesion and migration.

Invasion assays with PC3-GFP showed that $24 \mathrm{~h}$ pre-incubation with $1 \mu \mathrm{M}$ SIM did not affect invasion of PC3-GFP through Matrigel towards AA compared with control (Figure 5A, left panel). By contrast, invasion assays conducted with a confluent BMEC layer on Matrigel revealed that $24 \mathrm{~h}$ pre-incubation of PC3GFP with $1 \mu \mathrm{M}$ SIM decreased PC3-GFP TEM towards AA (Figure 5A, right panel). Incubation with $1 \mu \mathrm{M}$ SIM decreased both BMEC adhesion (Figure 5B) and AA-induced PC3-GFP 2D migration (Figure 5C) compared with the SIM-free controls.

Lovastatin has been shown to downregulate RhoA activity in squamous cell carcinoma (Zhao et al, 2010). Therefore, we tested the hypothesis that SIM might regulate this pathway in PC3-GFP cells. Rho assays showed that $1 \mu \mathrm{M}$ SIM reduced both PC3-GFP RhoA and RhoC activity over a $24-\mathrm{h}$ period (Figure 5D) and prevented the activation of both RhoA and RhoC by AA (Figure $5 \mathrm{E}$ ). At the same time, the addition of $1 \mu \mathrm{M}$ SIM reduced the levels of Akt and MLC2 phosphorylation induced by $10 \mu \mathrm{M} \mathrm{AA}$ in both PC3-GFP and DU-145 cells (Figure 5F-H). As observed in the PC-3 cell lines, $1 \mu \mathrm{M}$ SIM did not affect the ability of DU-145 to invade towards AA through a Matrigel barrier but it significantly reduced TEM across a BMEC layer (Figure 5I). The presence of SIM also reduced AA-induced DU-145 adhesion to BMECs and 2D migration assays (Figure 5J and K).

\section{DISCUSSION}

Motility and TEM abilities are a pre-requisite for metastatic spread of tumour cells. For this, cancer cells must possess the ability to reorganise their cytoskeleton. Cell migration has been classified according to morphological observation, which has since been associated with molecular analysis of cytoskeletal organisation and cellular interaction with the microenvironment (Friedl and Gilmour, 2009; Lammermann and Sixt, 2009; Sanz-Moreno and Marshall, 2009). In this study, we first analysed the invasive ability of different prostatic cell lines using TEM assays. We observed that only PC-3 cells and the sub-clone PC3-GFP were highly invasive, with DU-145 also being invasive but at a lower level than the previous two. Other cell lines used commonly in $\mathrm{CaP}$ experiments such as VCaP and LNCaP were unable to cross a confluent endothelium, confirming previous studies showing the differential invasive capacity of prostatic epithelial cells (Hart et al, 2005).

Initial observational studies demonstrated that an amoeboid phenotype was required by PC-3 cells for TEM across BMEC monolayers. In the presence of bone marrow conditioned media (Long Term Bone Marrow Media conditioned through 7 day contact with haematopoietically active, adipocyte-rich BMS) PC-3/ PC3-GFP cells adopted an amoeboid phenotype (Figure 2D; Supplementary Figure 2; Hart et al, 2005). The importance of this switch was confirmed by the inhibition of ROCK or by blebbistatin treatment, which decreased TEM. However inhibition of mesenchymal motility, mostly regulated by Rac signalling and the production of MMPs, did not change 3D migration across the bone marrow endothelium.

The amoeboid phenotype, facilitated by the Rho/ROCK signalling pathway, enables morphological changes which in turn enable epithelial cells to move through gaps in the ECM fibres or endothelium by adapting their morphology to pre-existing spaces (Sahai and Marshall, 2002). Rho in GTP-bound form activates its effector, Rho-associated serine-threonine protein kinase (ROCK), which then induces MLC2 phosphorylation (Kimura et al, 1996; Wilkinson et al, 2005). Once the MLC2 is phosphorylated (P-MLC2), the activity of myosin II ATPase is enhanced and myosin II becomes engaged in more efficient interactions with actin filaments, thus increasing cell contractility (Wilkinson et al, 2005).

To characterise the signalling mechanism behind BMS-induced TEM, it is necessary to break down the complex BMS environment and examine the role of individual components in TEM. In this study, we replaced human BMS with AA, a PUFA found in BM-ad; this is a powerful attractant for $\mathrm{CaP}$ cells and has been shown to have an important role in the metastasis-like behaviour in $\mathrm{CaP}$ in vitro (Brown et al, 2006, 2010; Gazi et al, 2007; Tawadros et al, 2012). AA induced rounding of the PC3-GFP cell morphology accompanied by an activation of the Rho/ROCK pathway. ROCK inhibition or specific silencing of RhoA, RhoC, ROCK1 and ROCK2 decreased TEM associated with AA without modulating invasion through a synthetic basement membrane.

We have shown previously that $\mathrm{CaP}$ cells bind to junctional areas of the BM endothelium (Hart et al, 2005) and we have consolidated these studies, conducting static adhesion experiments and performing assays within short time scales to mirror the rapidity of the process in vivo. In these we observed specific function of RhoA and ROCK2 but not RhoC and ROCK1 in AAinduced adhesion to BMEC (Figure 4A). Further studies are required to understand how that adhesion process is regulated by RhoA and ROCK2 with specific regard to integrins, in particular the $\beta 1$ integrin component: we have previously shown that this component is fundamental to the metastasis-like behaviour in $\mathrm{CaP}$ in vitro (Lang et al, 1997; Scott et al 2001; Hart et al, 2005). By contrast, migration in $2 \mathrm{D}$, a putative crucial step during TEM, is dependent on RhoC, ROCK1 and ROCK2. Finally, the results show that TEM is inducible and is clearly activated following AA stimulation.

Our studies also demonstrate specific elements controlling the functional mechanics of TEM. It has been reported previously that RhoA and RhoC have different functions in cell migration and invasion (Vega et al, 2011). This work showed that the amoeboid 
A

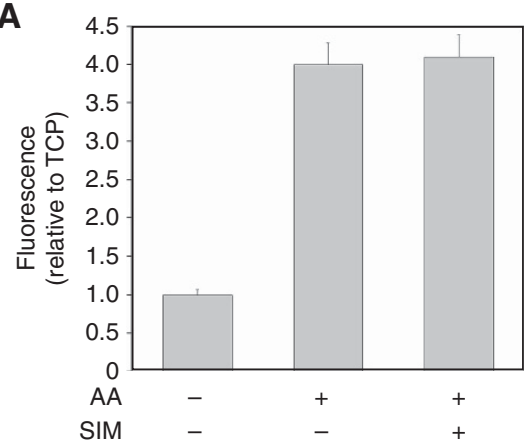

C

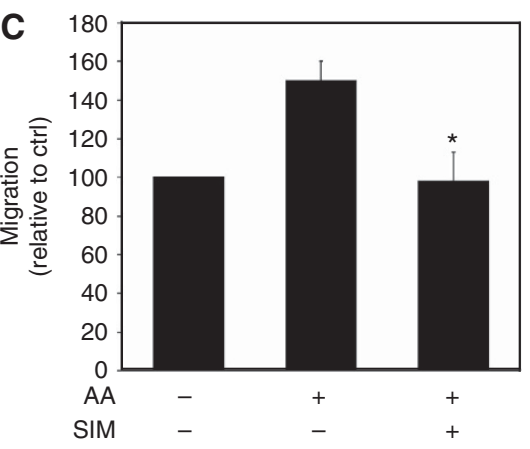

G

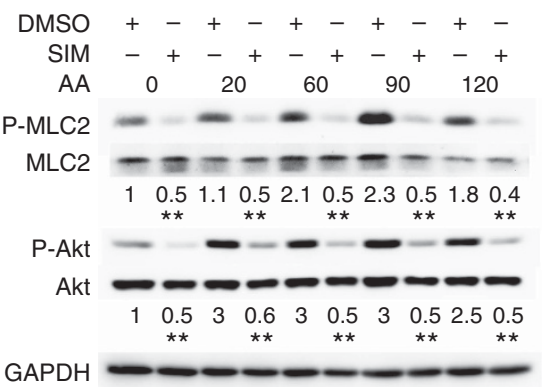

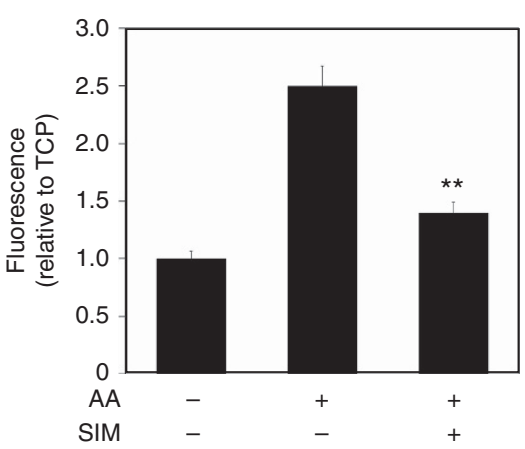

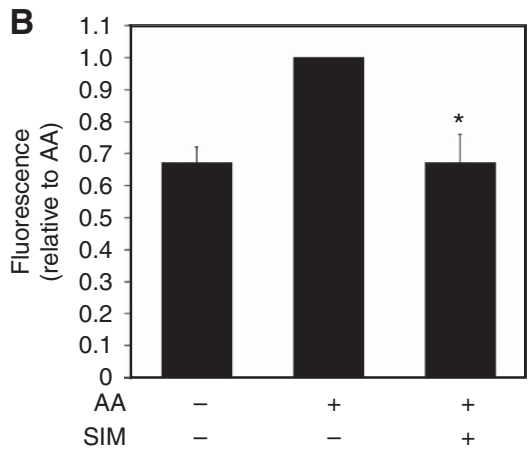

D

$\begin{array}{lllllll}\mathrm{SIM} & 0 & 1 & 2 & 6 & 12 & 24 \\ (\mathrm{~h})\end{array}$

GTP-RhoA - - . -

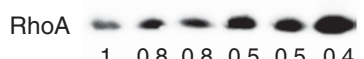

* $* \star * *$

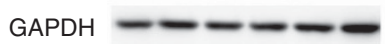

GTP-RhoC

RhoC

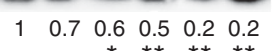

E

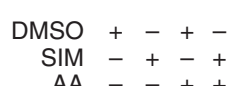

GTP-RhoA - - -

RhoA - - -

$10.6 \underset{\star}{0} \underset{* \star}{2} 0.2$

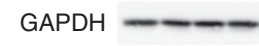

GTP-RhoC

RhoC

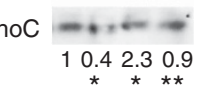

H

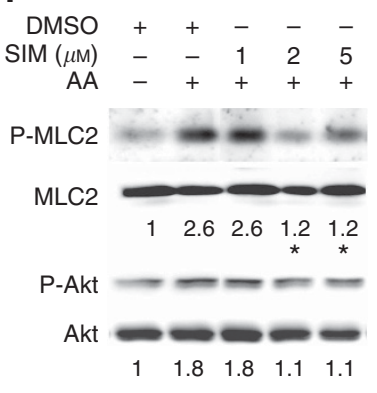

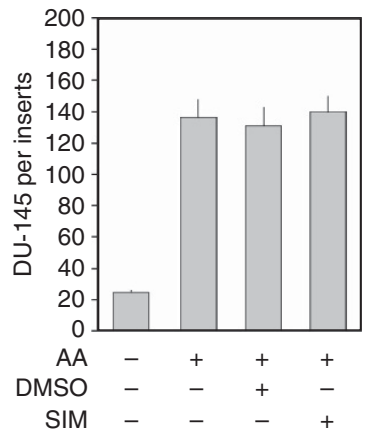

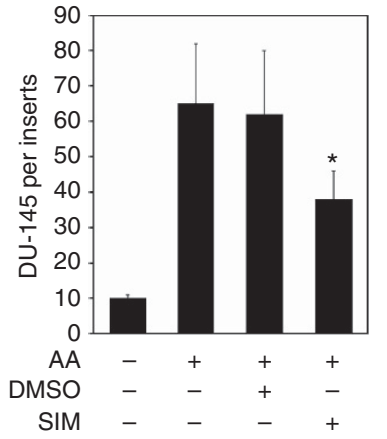

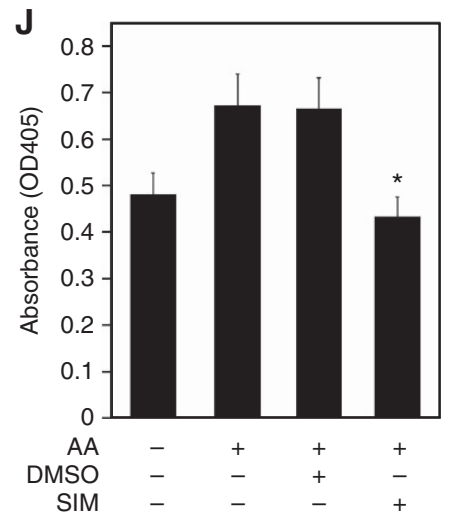

phenotype is dependent on RhoA, which increased invasion through Matrigel towards FCS. However, the pleiotropic effects of the chemotactic agent in FCS must be acknowledged. FCS is a mixture of cytokines, growth factor and lipids, all of which can stimulate various signalling pathways individually. In our study we focussed on a single chemotactic agent, enabling close observation of its individual effects, thereby facilitating specific analysis of the consequent downstream pathway activation. This has yielded new information but further work is required to find other factors

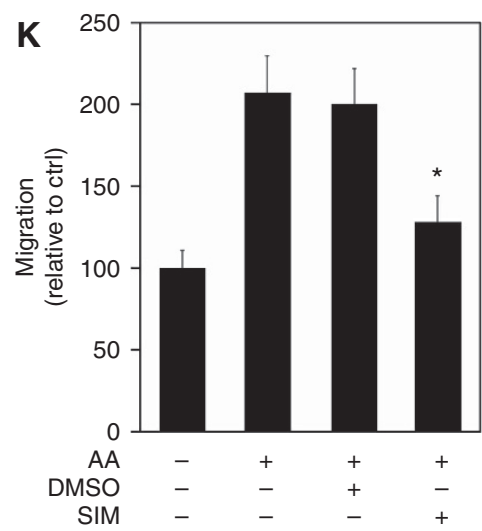

influencing RhoA, RhoC, ROCK1 and ROCK2 activity in CaP to understand their individual role in early implantation of circulating tumour cells in BMS.

We have previously shown that the lipophilic statins are potent inhibitors of metastasis-like behaviour of malignant prostate epithelial cells, working by inhibition of protein geranylgeranylation (Brown et al, 2012). Using SIM at concentrations validated in previous studies we confirmed that the statin acted on each individual step of the invasion pathway 
Figure 5. Statins decrease transendothelial migration through the Rho GTPase signalling pathway. (A) Invasion assays with PC3-GFP cells were performed using cell-culture inserts $(8 \mu \mathrm{m}$ pore size) coated by a synthetic basement membrane (Matrigel) (left panel) or culture inserts $(8 \mu \mathrm{m}$ pores) coated by a layer of BMEC above a synthetic basement membrane (Matrigel) (right panel). $2 \times 10^{5}$ PC3-GFP cells pre-incubated with $1 \mu \mathrm{M}$ simvastatin (SIM) or vehicle (DMSO) for $24 \mathrm{~h}$ were added to the top of the inserts and allowed to invade towards $\mathrm{AA}(10 \mu \mathrm{M})$ for $18 \mathrm{~h}$. Data represent means \pm s.e.m. of three separate experiments (in triplicate). ${ }^{\star} * P \leqslant 0.01$ vs no SIM towards AA. (B) Adhesion assays to BMEC \pm AA (10 $\mu \mathrm{M}$ ) were undertaken with PC3-GFP cells pre-incubated with $1 \mu \mathrm{M}$ SIM or vehicle only for $24 \mathrm{~h}$ before the assay. Data represent means $\pm \mathrm{s}$.e.m. of three separate experiments. ${ }^{*} \leqslant 0.05$ vs no SIM incubated with AA. (C) Migration assays were performed using PC3-GFP \pm AA (10 $\left.\mu \mathrm{M}\right)$. PC3-GFP cells were pre-incubated with $1 \mu \mathrm{M}$ SIM or vehicle only for $24 \mathrm{~h}$ before the assay. Data represent means \pm s.e.m. of three separate experiments. ${ }^{\star} P \leqslant 0.05$ vs vehicle only with AA. (D) Lysates of PC3-GFP cells incubated at different times with simvastatin $1 \mu \mathrm{m}(\mathrm{SIM})(0,1,2,6,12$ and $24 \mathrm{~h})$ were subjected to Rho GTPase assays. Levels of GTP-RhoA and GTP-RhoC were compared with total levels of RhoA and RhoC, respectively. Figure is representative of two separate experiments done in duplicate. ${ }^{*} P<0.05,{ }^{*} P<0.01$ vs no SIM. Densitometric values of controls without incubation with SIM (vehicle only) were set at one. (E) Lysates of PC3-GFP cells in \pm AA (10 $\mu \mathrm{M}) \pm$ pre-incubated with simvastatin $1 \mu \mathrm{M}(\mathrm{SIM})(24 \mathrm{~h})$ were subjected to Rho GTPase assays. Levels of GTP-RhoA and GTP-RhoC were compared with total levels of RhoA and RhoC, respectively. Figure is representative of two separate experiments done in duplicate. ${ }^{\star} P<0.05$ vs no SIM without $A A,{ }^{\star \star} P<0.01$ vs no SIM with $A A$. Densitometric values of controls without incubation with SIM (vehicle only)/AA were set at one. (F) P-MLC2 ${ }^{\text {t18/s } 19}$, MLC2, P-Akt and Akt content was analysed by western blotting in lysates of PC3-GFP cells incubated at different times with $1 \mu \mathrm{M}$ SIM or vehicle only (DMSO). Figure is representative of two separate experiments done in duplicate. ${ }^{\star} P<0.05,{ }^{*} P<0.01$ vs no SIM (vehicle only). Densitometric values of controls without incubation with SIM (vehicle only) were set at one. (G) P-MLC2 ${ }^{\mathrm{t} 18 / \mathrm{s} 19}, \mathrm{MLC2}, \mathrm{P}-\mathrm{Akt}^{\mathrm{s} 473}$ and Akt content was analysed by western blotting in lysates of PC3-GFP cells \pm preincubated with simvastatin $1 \mu \mathrm{M}(\mathrm{SIM})(24 \mathrm{~h})$ and incubated over time with $\mathrm{AA}(10 \mu \mathrm{M})$. Figure is representative of two separate experiments done in duplicate. ${ }^{*} P \leqslant 0.05$ vs Ctrl (DMSO) at the same time with AA incubation. Densitometric values of controls (DMSO, without AA) were set at one. (H) P-MLC2 ${ }^{\text {t18/s19 }}$, MLC2, P-Akt and Akt content was analysed by western blotting in lysates of DU-145 cells incubated at different times with AA $(10 \mu \mathrm{M})$. Levels of P-Akt and P-MLC2 were compared with total levels of Akt and MLC2, respectively. Figure is representative of two separate experiments done in duplicate. ${ }^{\star} P \leqslant 0.05$ vs Ctrl (DMSO) without AA. Densitometric values of controls were set at one. (I) Invasion assays with DU-145 cells were performed using cell-culture inserts ( $8 \mu \mathrm{m}$ pore size) coated by a synthetic basement membrane (Matrigel) (left panel) or culture inserts ( $8 \mu \mathrm{m}$ pores) coated by a layer of BMEC above a synthetic basement membrane (Matrigel) (right panel). $2 \times 10^{5} \mathrm{DU}-145 \mathrm{cells}$ were added to the top of the inserts and allowed to invade towards AA $(10 \mu \mathrm{m})$ for $18 \mathrm{~h}$. DU-145 cells were pre-incubated with SIM or vehicle only (DMSO) $24 \mathrm{~h}$ before the invasion assays. Data represent means \pm s.e.m. of three separate experiments (in triplicate). ${ }^{*} P<0.05$ vs DMSO towards AA. Cells were counted after staining with either crystal violet or CK immunostaining. (J) Adhesion assays to BMEC over 15 min $\pm A A(10 \mu \mathrm{M})$ were undertaken using DU-145 cells. These were pre-incubated with SIM or vehicle only (DMSO) $24 \mathrm{~h}$ before the adhesion assays. Data represent means \pm s.e.m. of three separate experiments. * $P \leqslant 0.05$ vs DMSO incubated with AA. (K) Migration assays were performed using DU-145 \pm AA (10 $\mu \mathrm{m})$. DU-145 cells were pre-incubated with SIM or vehicle only (DMSO) $24 \mathrm{~h}$ before the migration assays. Data represent means \pm s.e.m. of two separate experiments. ${ }^{\star} P \leqslant 0.05$ vs DMSO incubated with AA.

(adhesion, migration and TEM). Analysis of the regulating molecular pathways showed that Akt and Rho signalling in PC3GFP and DU-145 cells, which are known to be regulated by statins (Roy et al, 2011), was downregulated by SIM. Our previous work (Tawadros et al, 2012) demonstrated that the AA/Akt interaction occurred in lipid rafts, where cholesterol is an essential component. Lipid rafts are cellular membrane micro-domains that regulate signalling cascades originating from membrane bound receptors such as tyrosine kinases. Treatment of $\mathrm{CaP}$ with SIM is known to induce a $70 \%$ reduction in lipid raft cholesterol (Zhuang et al, 2005), and we have observed previously that Akt pathway activation by AA is dependent on lipid raft integrity (Tawadros et al, 2012). Thus, it is possible that the critical actions not only of AA but also of statins occur within the lipid rafts and that modification of the lipid composition of these could be critical to the metastatic process in CaP. However, it is also important to recognise that cholesterol-independent pathways mediating statin action are described (Roy et al, 2011) and these too may be influential.

The observed statin effect may be related, at least in part, to their effect on the G-proteins. Previously, we demonstrated that geranylgeranylation, but not farnesylation is a crucial element in lipophilic statin effect on the metastatic behaviour of $\mathrm{CaP}$ cells (Brown et al, 2012). The Rho and Rac subfamilies of small GTPase are mostly geranylgeranylated (Roy et al, 2011), which could explain the mechanism of amoeboid regulation by SIM seen in our study. It may also explain the effects of statins on prostate and other cancers in large population studies, where they are known to decrease $\mathrm{CaP}$ progression and mortality but not its incidence. Metastatic spread is the crucial process in cancer mortality and targeting cellular motility and endothelial transmigration mechanisms associated with TEM may have a significant therapeutic potential. Our observations show the importance of G-proteins in cancer cell migration/motility and endothelial trans-migration and provide further evidence that lipophilic statins may influence this process.

In conclusion, the data presented herein show that the Rho signalling is involved in TEM in prostatic cancer in vitro. AA and SIM, two putative modulators of $\mathrm{CaP}$ aggressiveness, are able to alter this process significantly. TEM is an important potential therapeutic target for limiting $\mathrm{CaP}$ progression and metastasis. New knowledge regarding the role of fatty acid metabolism may facilitate this development.

\section{ACKNOWLEDGEMENTS}

This work was supported by grants from the Swiss National Science Foundation: 'Bourse chercheur debutant' (PBLAP3129433/1) and 'Bourse pour chercheur avance aupres FSBMB/ Novartis' (PASMP3_134378/1); and by supporting funds from the GU Cancer Research Group endowments, including the Christie Hospital and the Christie based 'Men Matter' Charity.

\section{CONFLICT OF INTEREST}

The authors declare no conflict of interest.

\section{REFERENCES}

Aktories K, Just I (2005) Clostridial Rho-inhibiting protein toxins. Curr Top Microbiol Immunol 291: 113-1145.

Boureux A, Vignal E, Faure S, Fort P (2007) Evolution of the Rho family of ras-like GTPases in eukaryotes. Mol Biol Evol 24(1): 203-216. 
Breau RH, Karnes RJ, Jacobson DJ, McGree ME, Jacobsen SJ, Nehra A, Lieber St MM, Sauver JL (2010) The association between statin use and the diagnosis of prostate cancer in a population based cohort. J Urol 184(2): 494-499.

Brown M, Hart C, Tawadros T, Ramani V, Sangar V, Lau M, Clarke N (2012) The differential effects of statins on the metastatic behaviour of prostate cancer. Br J Cancer 106(10): 1689-1696.

Brown MD, Hart C, Gazi E, Gardner P, Lockyer N, Clarke N (2010) Influence of omega-6 PUFA arachidonic acid and bone marrow adipocytes on metastatic spread from prostate cancer. Br J Cancer 102(2): 403-413.

Brown MD, Hart CA, Gazi E, Bagley S, Clarke NW (2006) Promotion of prostatic metastatic migration towards human bone marrow stoma by Omega 6 and its inhibition by Omega 3 PUFAs. Br J Cancer 94(6): 842-853.

Chavarro JE, Stampfer MJ, Li H, Campos H, Kurth T, Ma J (2007) A prospective study of polyunsaturated fatty acid levels in blood and prostate cancer risk. Cancer Epidemiol Biomarkers Prev 16(7): 1364-1370.

Dietrich KA, Schwarz R, Liska M, Grass S, Menke A, Meister M, Kierschke G, Langle C, Genze F, Giehl K (2009) Specific induction of migration and invasion of pancreatic carcinoma cells by RhoC, which differs from RhoA in its localisation and activity. Biol Chem 390(10): 1063-1077.

Friedl P, Gilmour D (2009) Collective cell migration in morphogenesis, regeneration and cancer. Nat Rev Mol Cell Biol 10(7): 445-457.

Gazi E, Gardner P, Lockyer NP, Hart CA, Brown MD, Clarke NW (2007) Direct evidence of lipid translocation between adipocytes and prostate cancer cells with imaging FTIR microspectroscopy. J Lipid Res 48(8): 1846-1856.

Gutt R, Tonlaar N, Kunnavakkam R, Karrison T, Weichselbaum RR, Liauw SL (2010) Statin use and risk of prostate cancer recurrence in men treated with radiation therapy. J Clin Oncol 28(16): 2653-2659.

Hart CA, Brown M, Bagley S, Sharrard M, Clarke NW (2005) Invasive characteristics of human prostatic epithelial cells: understanding the metastatic process. Br J Cancer 92(3): 503-512.

Jaffe AB, Hall A (2005) Rho GTPases: biochemistry and biology. Annu Rev Cell Dev Biol 21: 247-269.

Kimura K, Ito M, Amano M, Chihara K, Fukata Y, Nakafuku M, Yamamori B, Feng J, Nakano T, Okawa K, Iwamatsu A, Kaibuchi K (1996) Regulation of myosin phosphatase by Rho and Rho-associated kinase (Rho-kinase). Science 273(5272): 245-248.

Kitzing TM, Wang Y, Pertz O, Copeland JW, Grosse R (2010) Formin-like 2 drives amoeboid invasive cell motility downstream of RhoC. Oncogene 29(16): 2441-2448.

Kopfstein L, Christofori G (2006) Metastasis: cell-autonomous mechanisms versus contributions by the tumor microenvironment. Cell Mol Life Sci 63(4): 449-468.

Lammermann T, Sixt M (2009) Mechanical modes of 'amoeboid' cell migration. Curr Opin Cell Biol 21(5): 636-644.

Lang SH, Clarke NW, George NJ, Allen TD, Testa NG (1998) Interaction of prostate epithelial cells from benign and malignant tumor tissue with bone-marrow stroma. Prostate 34(3): 203-213.

Lang SH, Clarke NW, George NJ, Testa NG (1997) Primary prostatic epithelial cell binding to human bone marrow stroma and the role of alpha2beta1 integrin. Clin Exp Metastasis 15(3): 218-227.

Leung T, Chen XQ, Manser E, Lim L (1996) The p160 RhoA-binding kinase ROK alpha is a member of a kinase family and is involved in the reorganization of the cytoskeleton. Mol Cell Biol 16(10): 5313-5327.

Loberg RD, Logothetis CJ, Keller ET, Pienta KJ (2005) Pathogenesis and treatment of prostate cancer bone metastases: targeting the lethal phenotype. J Clin Oncol 23(32): 8232-8241.
Loeb S, Kan D, Helfand BT, Nadler RB, Catalona WJ (2010) Is statin use associated with prostate cancer aggressiveness? BJU Int 105(9): 1222-1225.

Murtola TJ, Tammela TL, Maattanen L, Huhtala H, Platz EA, Ala-Opas M, Stenman UH, Auvinen A (2010) Prostate cancer and PSA among statin users in the Finnish prostate cancer screening trial. Int J Cancer 127(7): $1650-1659$.

Pankova K, Rosel D, Novotny M, Brabek J (2010) The molecular mechanisms of transition between mesenchymal and amoeboid invasiveness in tumor cells. Cell Mol Life Sci 67(1): 63-71.

Platz EA (2008) Re: The association between statins and cancer incidence in a veterans population. J Natl Cancer Inst 100(13): 972; author reply 973-974.

Platz EA, Leitzmann MF, Visvanathan K, Rimm EB, Stampfer MJ, Willett WC, Giovannucci E (2006) Statin drugs and risk of advanced prostate cancer. J Natl Cancer Inst 98(24): 1819-1825.

Roy M, Kung HJ, Ghosh PM (2011) Statins and prostate cancer: role of cholesterol inhibition vs. prevention of small GTP-binding proteins. Am J Cancer Res 1(4): 542-561.

Sahai E, Marshall CJ (2002) RHO-GTPases and cancer. Nat Rev Cancer 2(2): 133-142.

Sanz-Moreno V, Marshall CJ (2009) Rho-GTPase signaling drives melanoma cell plasticity. Cell Cycle 8(10): 1484-1487.

Schumacher MC, Laven B, Petersson F, Cederholm T, Onelov E, Ekman P, Brendler C (2011) A comparative study of tissue omega- 6 and omega-3 polyunsaturated fatty acids (PUFA) in benign and malignant pathologic stage pT2a radical prostatectomy specimens. Urol Oncol 31(3): 318-324.

Scott LJ, Clarke NW, George NJ, Shanks JH, Testa NG, Lang SH (2001) Interactions of human prostatic epithelial cells with bone marrow endothelium: binding and invasion. Br J Cancer 84(10): 1417-1423.

Szymanski KM, Wheeler DC, Mucci LA (2010) Fish consumption and prostate cancer risk: a review and meta-analysis. Am J Clin Nutr 92(5): 1223-1233.

Tawadros T, Brown MD, Hart CA, Clarke NW (2012) Ligand-independent activation of EphA2 by arachidonic acid induces metastasis-like behaviour in prostate cancer cells. Br J Cancer 107(10): 1737-1744.

Thiery JP (2002) Epithelial-mesenchymal transitions in tumour progression. Nat Rev Cancer 2(6): 442-454.

Vega FM, Fruhwirth G, Ng T, Ridley AJ (2011) RhoA and RhoC have distinct roles in migration and invasion by acting through different targets. J Cell Biol 193(4): 655-665.

Wheeler AP, Ridley AJ (2004) Why three Rho proteins? RhoA, RhoB, RhoC, and cell motility. Exp Cell Res 301(1): 43-49.

Wilkinson S, Paterson HF, Marshall CJ (2005) Cdc42-MRCK and Rho-ROCK signalling cooperate in myosin phosphorylation and cell invasion. Nat Cell Biol 7(3): 255-261.

Wynder EL, Rose DP, Cohen LA (1994) Nutrition and prostate cancer: a proposal for dietary intervention. Nutr Cancer 22(1): 1-10.

Zhao TT, Le Francois BG, Goss G, Ding K, Bradbury PA, Dimitroulakos J (2010) Lovastatin inhibits EGFR dimerization and AKT activation in squamous cell carcinoma cells: potential regulation by targeting rho proteins. Oncogene 29(33): 4682-4692.

Zhuang L, Kim J, Adam RM, Solomon KR, Freeman MR (2005) Cholesterol targeting alters lipid raft composition and cell survival in prostate cancer cells and xenografts. J Clin Invest 115(4): 959-968.

This work is published under the standard license to publish agreement. After 12 months the work will become freely available and the license terms will switch to a Creative Commons AttributionNonCommercial-Share Alike 3.0 Unported License.

Supplementary Information accompanies this paper on British Journal of Cancer website (http://www.nature.com/bjc) 